Technological University Dublin

DÜBLIN

ARROW@TU Dublin

1995

\title{
Degradation of Optical Bistability in Surface Stabilized Ferroelectric Liquid Crystal (SSFLC) Cells.
}

\author{
S. Mac Lughadha \\ Trinity College Dublin, Ireland \\ Yuri Panarin \\ Technological University Dublin, yuri.panarin@tudublin.ie \\ J. Vij \\ Trinity College Dublin, Ireland
}

Follow this and additional works at: https://arrow.tudublin.ie/engscheleart2

\section{Recommended Citation}

Vij, J., Panarin, Y. \& Mac Lughadha, S. (1995) Degradation of Optical Bistability in Surface Stabilized Ferroelectric Liquid Crystal (SSFLC) Cells, Mathematica Aeterna, Vol. 263, pp.169-177. 1995. DOI: $10.1080 / 10587259508033581$

This Article is brought to you for free and open access by the School of Electrical and Electronic Engineering at ARROW@TU Dublin. It has been accepted for inclusion in Articles by an authorized administrator of ARROW@TU Dublin. For more information, please contact arrow.admin@tudublin.ie, aisling.coyne@tudublin.ie, gerard.connolly@tudublin.ie.

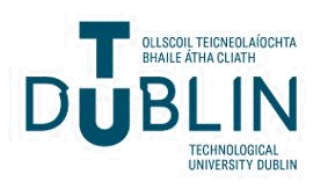


Mol. Cryst. Liq. Cryst. 1995, Vol. 263, pp. 169-177 Reprints available directly from the publisher Photocopying permitted by license only
(C) 1995 OPA (Overseas Publishers Association) Amsterdam B. V. Published under license by Gordon and Breach Science Publishers SA Printed in Malaysia

\title{
DEGRADATION OF OPTICAL BISTABILITY IN SURFACE STABILIZED FERROELECTRIC LIQLID CRYSTAL (SSFLC) CELLS
}

\author{
S.T.MAC LUGHADHA, YU.P.PANARIN $\times$ and J.K.VIJ $^{+}$ \\ Department of Microelectronics and Electrical Engineering, University \\ of Dublin, Trinity College, Dublin 2, Ireland. \\ X Organic Intermediates \& Dyes Institute, B.Sadovaya, 1/4, Moscow, \\ 103787, Russia.
}

\begin{abstract}
We investigate the degradation of optical bistability (bistability sticking) in FLC cells with alignment layers of different thicknesses using the static optical hysteresis technique. By storing these cells with and without DC field across the cell and by measuring the subsequent offset of the hysteresis curve we find that all cells exhibit bistability sticking. We find that the degradation of bistability is due not only to ionic effects but could also be due to an additional effect which is independent of the thickness of the alignment layer.
\end{abstract}

\section{$\underline{\text { INTRODUCTION }}$}

Although the first FLC television screen was developed ${ }^{1}$ in 1985 it is only recently that the commercial production of displays of this kind has begun. This arises from the complex nature of FLC's which hinders the practical application of such potentially powerful materials. It has been found that the most difficult problem preventing the practical application of these materials in highly multiplexed screens is the degradation of the optical bistability. This is exhibited as the shadow like reappearance 2 of a previously stored image when the device is switched on. The conventional explanation for the degradation of bistability in FLC displays is the build up of ions at the electrodes due to the spontaneous polarization field itself 1,2 and this field is proportional to the alignment layer thickness ${ }^{3}$. However we have found that bistability sticking also exists in FLC cells that are prepared without alignment layer (AL). This fact shows that the degradation of bistability is not only due to ionic effects but could also be due to an additional effect that is independent of alignment layer thickness (d'). 
However, degradation of the optical bistability in FLC's has been mentioned in very few publications 4,5 and has not yet been thoroughly investigated. To overcome this problem one should normally use FLC materials of low spontaneous polarization $\left(<5-10 \mathrm{nC} / \mathrm{cm}^{2}\right)$, very thin or conducting aligning layers and/or materials with a negative dielectric anisotropy.

It is on account of these considerations that there exists a need for detailed investigations into the problems of bistability degradation (sticking) with respect to the cell parameters such as the spontaneous polarization. cell thickness and alignment layer, etc. In this paper we provide evidence for the existence of at least two processes that are responsible for the bistability sticking.

\section{EXPERIMENTAL DETAILS}

Static hysteresis 6 is found to be a powerful technique for describing bistability and for investigating the bistability properties of FLC's. We use this simple technique to investigate the bistability of FLC cells by measuring the offset of the optical hysteresis curve. In the experiment we use cells of $4 \mu \mathrm{m}$ cell thickness prepared using glass with conducting electrodes. These are spin coated with PVA of various thicknesses. The liquid crystal mixture, SCE13, was introduced into the cells by capillary action in the isotropic phase. Uniform bookshelf structure was obtained by electric field treatment $(40 \mathrm{~V} / \mu \mathrm{m})$ in the chiral smectic $\mathrm{C}\left(\mathrm{SmC}^{*}\right)$ phase. All cells prior to experiments possess bistability, that is the centre of the hysteresis curve is at zero volts. Hysteresis curves were obtained by applying a triangular voltage $(3 \mathrm{~V}$ at $0.02 \mathrm{~Hz})$ to the cell and recording the transmittance over one period.

Since SSFLC devices are essentially dc field driven, it is therefore interesting to investigate the evolution of the hysteresis curve after the FLC cell has been subjected to bias field conditions. We use a series of measurements by storing the FLC cells with and without dc fields to try and identify the nature of the processes which are responsible for the degradation of optical bistability of FLC devices.

\section{RESULTS AND DISCUSSION}

Initially the cell is stored short-circuited in one of its optically switched states. Periodically the sample is reconnected to the alternating voltage and the hysteresis 
curve is obtained over a single cycle of the triangular waveform. The cell is again short-circuited in the previously stored state. Shown in Fig. 1(a-c) are the hysteresis curves for a cell before and after storing in one of its states. As can be clearly seen initially the cell is bistable and the hysteresis curve is shown to have its centre at zero.

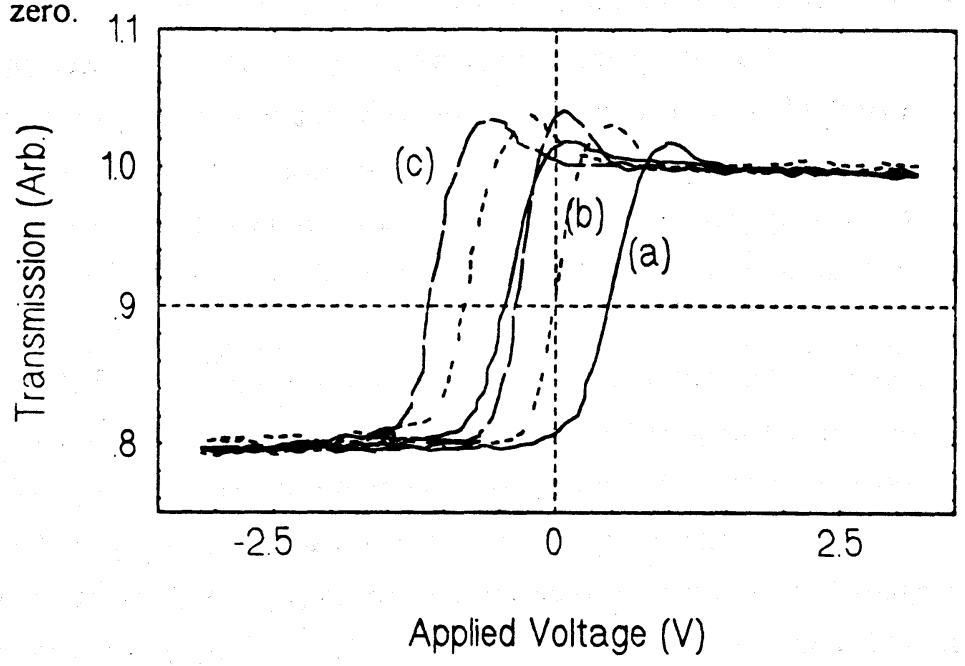

Fig.1 Optical hysteresis curves recorded before and after the cell has been stored in one of its optically switched states. (a) Initially bistable cell after (b) 30 and (c) 500 minutes storing short-circuited.

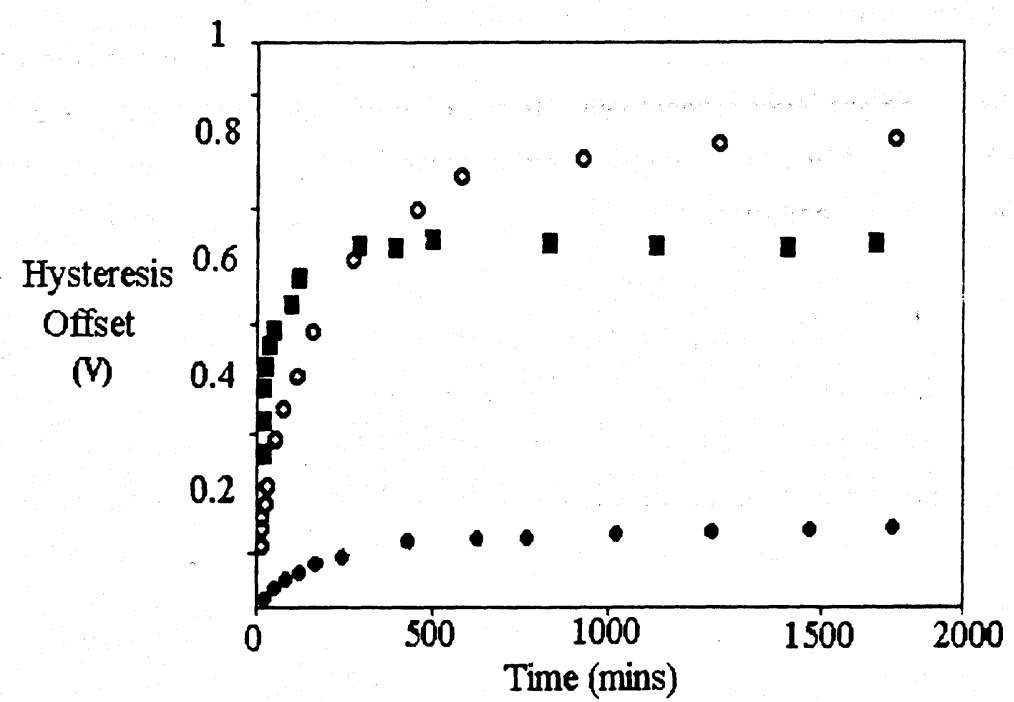

Fig. 2 Offset of the centre of the optical hysteresis curves as a function of 
time for cells with different $\mathrm{PVA}$ alignment layer thickness. At 0 mins the cells were short-circuited. ( $-640 \AA, \bigcirc-160 \AA, \bullet-0 \AA)$

During short-circuiting of the cells the stored optical switched state becomes more stable than the opposite state and this leads to a shift of the centre of the optical hysteresis loop along the voltage axis (Fig. 1). It can be seen in Fig. 2 that the offset of the hysteresis curve increases with time until a saturation value is achieved. This effect can be seen to occur for samples both with and without aligning layer. The degradation of bistability may be seen in the way that the curves recorded at later times have their centres shifted along the horizontal voltage axis

The common explanation for the degradation of bistability in FLC displays is the separation of the ions to the electrodes due to electric field created by the spontaneous polarization ${ }^{1,2}$. According to Yang et al ${ }^{3}$ the electric field inside the cell is a sum of the field induced by the applied external voltage $V_{\text {ext }}$ the field due to the free charges distributed between the electrodes, $\sigma_{S}$, and the spontaneous polarization, $\sigma_{p}$, and can be expressed as follows:

$$
E(t)=\frac{V_{e x t} \cdot \varepsilon^{\prime}+2 \sigma_{s} d^{\prime}+2 \sigma_{p} d^{\prime}}{2 d^{\prime} \varepsilon+d \varepsilon^{\prime}}
$$

where $\varepsilon, \varepsilon^{\prime}$ and $\mathrm{d}, \mathrm{d}^{\prime}$ are the dielectric permittivity and the thickness of the FLC cell and the alignment layer respectively. In the case of uniform states $\sigma_{p}= \pm P_{s}$. For describing the free charge distribution process for FLC cells one can apply the simple continuity equation:

$$
\frac{\partial n}{\partial t}=-\frac{n(t) \cdot E(t) \cdot \mu}{d}
$$

where $n$ and $\mu$ are the concentration and the mobility of free charges respectively. Thus by short-circuiting the cell the ions will spread away to the electrodes due to the electric field:

$$
E(t)=\frac{2 d^{\prime}\left(P_{s}-\sigma_{s}\right)}{2 d^{\prime} \varepsilon+d \varepsilon^{\prime}}
$$


until $\sigma_{S}=P_{\mathrm{S}}$ or $\sigma_{S}=n_{O} d$. This process can be schematically represented by Fig. 3 .

(a)

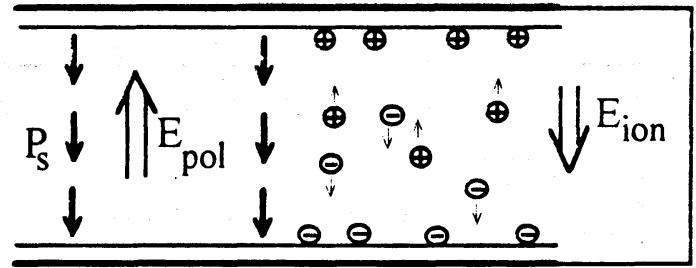

Figure 3. Schematic representation of the ionic polarization process which occurs during the short-circuiting of an FLC cell.

The field produced by ions $E_{i o n}$ is parallel to the spontaneous polarization vector $\vec{P}=P_{\boldsymbol{S}} / P_{S}$ making this state more stable than opposite one. According to conventional considerations the field produced by the ions is proportional to the alignment layer thickness 3 and therefore the phenomena of bistability sticking should be absent in FLC cells that possess either no alignment layer or conductive alignment lavers

We can see from Fig. 2 that bistability sticking occurs also in cells prepared without alignment layer. This contradiction between the conventional model for bistability degradation and experiment demonstrates that the degradation of bistability may not be simply explained by the generation of a depolarization field due to charge injection at the LC-AL interface.

When this procedure is complete an alternating triangular wave voltage $(3 \mathrm{~V}$ $p-p, 0.02 \mathrm{~Hz}$ ) is then constantly applied to the cells and the offset of the hysteresis curve with time may again be monitored. It may be seen from Fig. 4 that the offset of the hysteresis for cells both with and without alignment layers goes directly to zero. Figure 4 shows that under this alternating voltage that the cells return directly to their initial bistable conditions, and this also involves one process (ie. the offset of the centre of the hysteresis curves returns to zero volts).

The most striking contradiction with the ionisation model for FLC's has been found from the following experiment. We stored samples with various thicknesses of alignment layer for one hour under a direct voltage (30V) to ensure polarization of one switched state. After this procedure the stored state should become unstable due to ionic effects 4,5 . This can be seen as a shift of the centre 
of the hysteresis curve in the positive direction (Fig.5) and may be explained by a simple inverted bistability model (Fig.6).

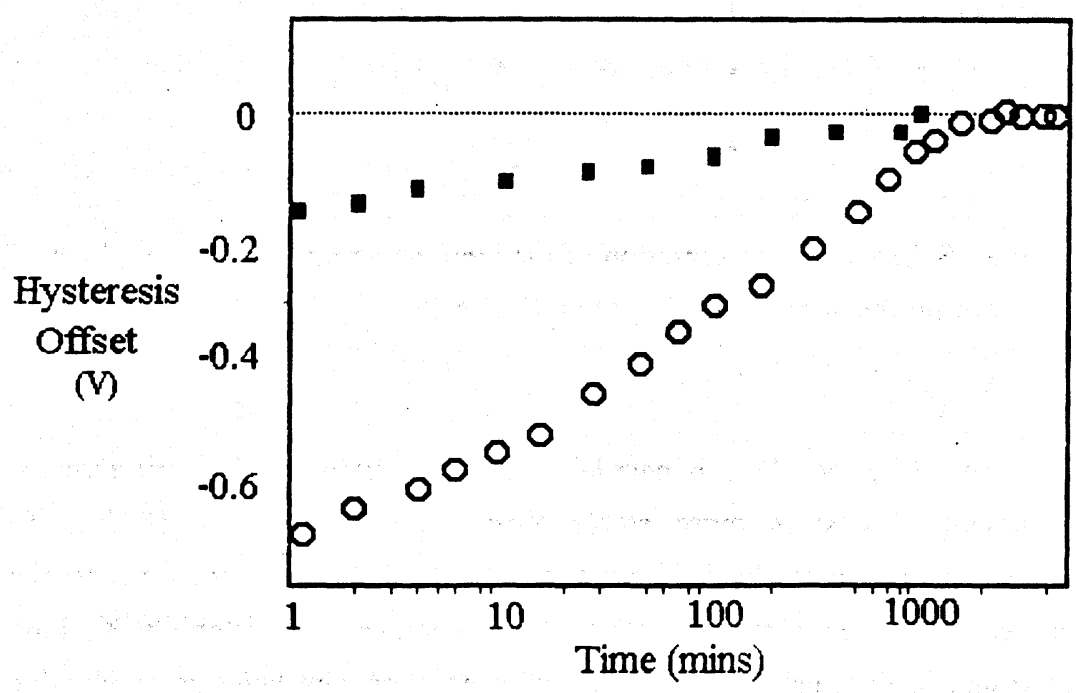

Fig.4 Offset of the centre of the optical hysteresis curves as a function of time (log scale) for cells with PVA alignment layer thickness under an alternating triangular voltage after short circuiting.( $-0 \AA, \bigcirc-160 \AA)$

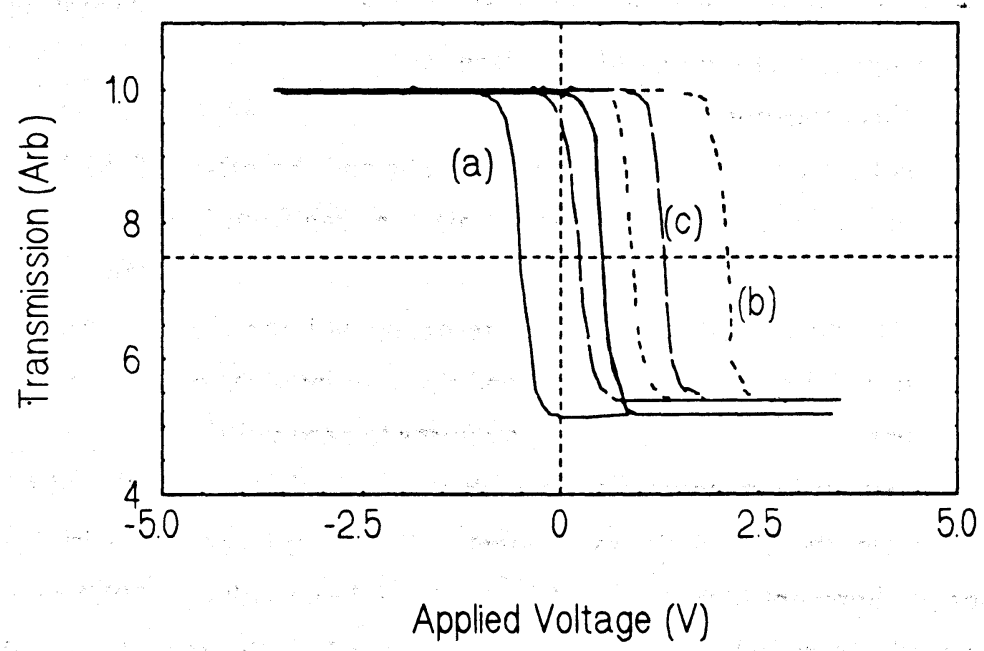

Fig. 5 Optical hysteresis curves for (a) initially bistable cell (b) after treatment with $30 \mathrm{~V} \mathrm{dc}$ (c) 30 mins after reconnecting to external alternating triangular waveform. 
Application of a high bias voltage to the cell leads to the accumulation of charges of opposing signs on the cell electrodes. If the resulting ionic field is sufficiently large then on removal of the external voltage a reversal of the director will occur and the cell will exhibit monostability in the state opposite to the original stored state. It is interesting to note that FLC cells with quite thick alignment layer

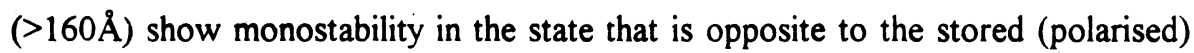
state. This is in good agreement with the ionic transport model for FLC's mentioned above. It should be noted here that this inversion of optical bistability did not occur for cells which did not possess alignment layer (ie. for cells with no aligning layer the stored state remained stable after the application of the dc voltage).

(a)

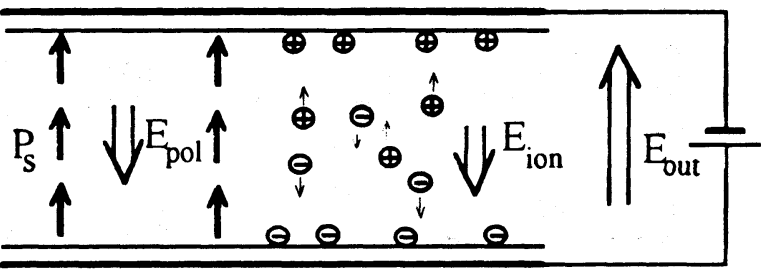

(b)

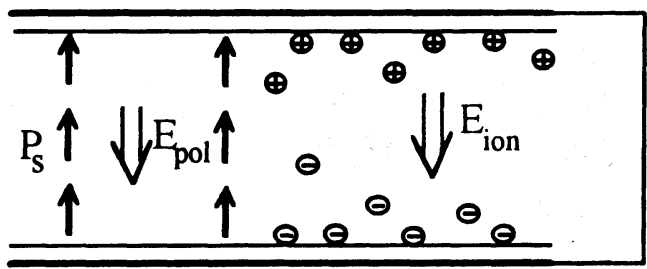

Fig.6 The inverted bistability model for a SSFLC Cell with alignment layer:

(a) On the application of a unipolar applied external field a build up of $_{h}$ ions on the electrodes occurs. (b) On the removal of this external voltage if the induced ionic field is sufficiently large an inversion of the optical bistability occurs which may ultimately lead to mono-stability of the cell.

According to the ionic transport model in FLC's $4-6$ on the application of an alternating field this polarisation effect should disappear after a certain time and the cell will return to its initial bistable condition. It can be clearly seen from Fig.7 that for cells with alignment layer $(\mathbb{D})$ that the hysteresis shift does not go to zero but passes through zero to a finite value in the opposite direction. The cell 
becomes monostable in the opposite state and only after this does it become bistable again.

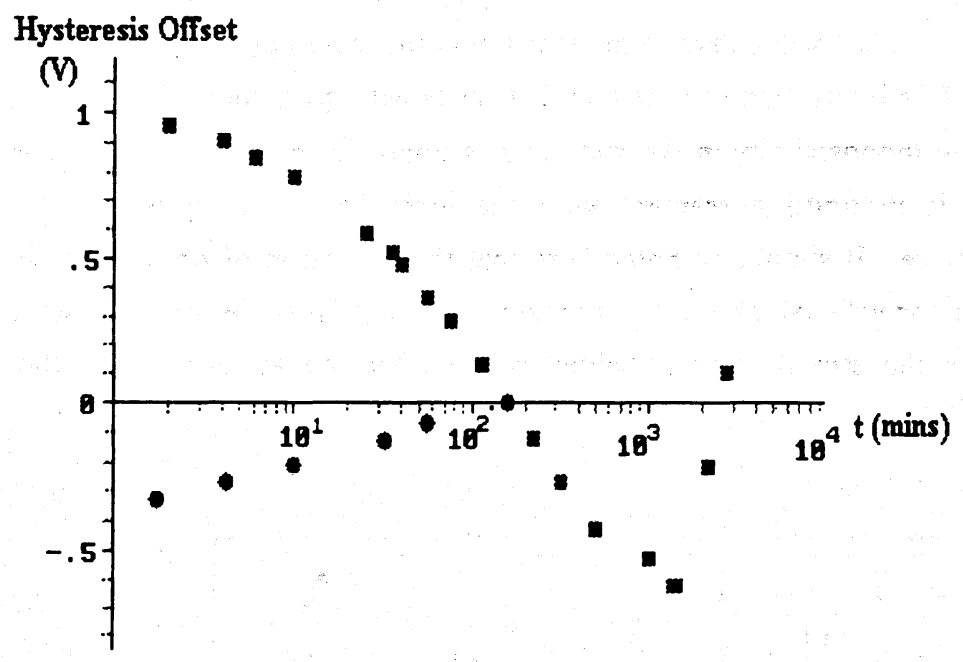

Figure 7. Offset of the centre of the hysteresis loop with time after polarization with $\mathrm{DC}$ field $(30 \mathrm{~V})$ of bright state for cells of different orientant layer (PVA) thickness. ( $\left(\mathrm{d}=4 \mu \mathrm{m}, \mathrm{P}_{\mathrm{s}}=27 \mathrm{nC} / \mathrm{cm}^{2}\right)$

- $\mathrm{d}^{\prime}=160 \AA$ This cell clearly exhibits two opposing relaxation processes .

- $\mathrm{d}^{\prime}=0 \AA$ (ie. no alignment layer) This cell exhibits only one process.

For the cell without the alignment layer only one depolarization process $(\bullet)$ is seen to occur and the cell returns directly to its initial bistable condition. It is clear that bistability degradation could be explained by the existence of two processes one due to ionic effects and another which is independent of alignment layer thickness and in the opposite direction to the ionic process.

\section{CONCLUSION}

On the application of an alternating field to the cells, the ionic transport model in FLC's explains the disappearance of any polarization effects after time and the resulting return of the FLC cell to its initial bistable condition. We find that for cells with alignment layer the offset of the hysteresis curve does not go to zero but 
through zero to a finite value with the cell becoming monostable in the opposite state only after this does the cell become bistable again. For the cell without alignment layer there is only one depolarization process and the cell returns directly to its initial bistable condition.

To summarise the experimental data we note that there are obviously two different processes responsible for bistability sticking in FLC cells. The first is in good agreement with the ionic model due to the electric field inside a cell with alignment layer and a second one which exists in all cells and is responsible for a reversal of the hysteresis offset.

The nature of this second process is not known and there is a definite need for a detailed investigation of this effect. The appropriate choice of cell parameters could eliminate the effect of both processes. This should facilitate the use of FLC's with high spontaneous polarization with uniform bookshelf structure with an increase in the multiplexing ratios of displays.

\section{ACKNOWLEDGEMENTS}

The authors would like to thank T.Begley for his assistance in carrying out the measurements.

\section{REFERENCES.}

1. T.Harada, M.Taguchi, K.Iwasa, M.Kai. SID 85 Digest, 131 (1985)

2. S.T.Lagerwall. N.Clark, J.Dijon, J.F.Clerc. Ferroelectrics, 94, 3-62 (1989).

3. K.H.Yang, T.C.Chieu, Jap.J.Appl.Phys., 28(9), L1599 (1989) and K.H.Yang, T.C.Chieu, S.Osofsky. Appl.Phys.Lett., 55(2), 125 (1989)

4. Y.Inaba, K.Katagiri, H.Inoue, Ferroelectrics 85, 255-264 (1988)

5. Z.Zou, N.A.Clark, M.A.Handschy, Ferroelectrics, 121, 147 (1991)

6. Yu.P.Panarin., Mol.Mat., 2(2),137 (1993) 